\title{
Duration, luminance, and the brightness exponent
}

\author{
S. S. STEVENS \\ LABORATORY OF PSYCHOPHYSICS, HARVARD UNIVERSITY
}

The relation of brightness to duration and luminance has been studied by matching one brightness to another and also by matching numbers to brightnes ses (magnitude estimation). The two methods concur in confirming certain well-known visual functions: Bloch's law, the Broca-Sulzer effect, and the shift of the Broca-Sulzer enhancement to shorter durations when luminance increases. It is shown that the shift with luminance requires the exponent of the power function for short-flash brightness to be larger than the exponent for stimuli of longer duration. An attempt is made to arlalyze some of the reasons why the procedure advocated by Graham may not give comparable results.

The dependence of apparent brightness on the duration of a flash has been a subject of such sustained interest in visual research that several methods have been employed to study the phenomenon. Bills (1920) reviewed the extensive work on the problem prior to 1920 . Recent experiments have used one or the other of two principal procedures, both involving a matching or balancing process. Under one procedure, the observer matches two brightnesses, e.g., that of a short flash to that of a long flash (Katz, 1964; Nachmias \& Steinman, 1965). Under the other procedure, the observer matches numbers to brightnesses. Other cross-modality matches are also possible, but thus far the role of flash duration in the determination of brightness has not been studied by matching, say, loudness to brightness. Loudnessbrightness matches have, however, been successfully made to verify the relation between the exponents of the power functions governing loudness and brightness (J. C. Stevens \& Marks, 1965).

Matches between number and brightness can be carried out in many different ways. One procedure used in the study of brief flashes (Raab, 1962) has produced results that accord well with the matches made directly between brightnesses. Another procedure of number matching, a method proposed by $\operatorname{Graham}(1965$, p. 73), has had a less fortunate outcome in the study reported by Lewis (1965). An attempt will be made below to analyze some of the difficulties with this second procedure. First, however, it is of interest to compare the results of brightness matching with those of number matching.

\section{Matching Brightness to Brightness}

In order to study the relation of brightness to duration and luminance, an effort was made by Aiba and Stevens (1964) to explore a wide range of stimulus durations and flash luminances. In one part of the experiment, the observer adjusted a standard 1-sec. flash, seen by one eye, to match the brightness of various short flashes, seen by the other eye. In another part of the experiment, the observer adjusted the shorter flash to balance its brightness against that of the longer flash. It is interesting that the results show a slight, but systematic, difference depending on which stimulus is adjusted by the observer. The phenomenon, often called the regression effect, seems to occur because the observer tends to constrict the range of whichever variable he has under his control (Stevens, 1959). Whatever the exact cause, the ubiquity of the regression effect suggests that it may be desirable, whenever possible, to counterbalance the experimental procedure by making matches in both directions.

The results shown in Fig. 1 are those obtained by Aiba and Stevens for five observers, each of whom made four adjustments of each stimulus. The filled points are geometric means of the 20 judgments; the unfilled points are medians. The curves fitted to the data represent an attempt to satisfy the requirement imposed by Bloch's law, namely, that over the shorter durations the slopes in each plot mustbe approximately equal to one and the spacing approximately uniform. The ability of the curves to satisfy that requirement shows that Bloch's law holds at suprathreshold levels: for a given brightness of flash, duration can be traded for luminance.

An obvious feature of the curves in Fig. 1 is the brightness enhancement (Broca-Sulzer effect) that occurs at durations between about 30 and $300 \mathrm{msec}$. As indicated by the dashed lines, the duration at which the maximum brightness occurs tends to decrease systematically as the luminance of the flash is increased. The shift in the position of the Broca-Sulzer hump, indicated by the dashed lines in Fig. 1, has an interesting implication concerning the exponent of the power function relating brightness to luminance. This point is discussed below.

\section{Matching Numbers to Brightmess}

Under the simple instruction to assign numbers to stimuli in such a way that the numbers are proportional to the apparent intensity of the stimuli, observers have scaled subjective intensity in numerous sense modalities. The procedure, often called magnitude estimation, has revealed a basic invariance in the form of the operating characteristics of the sensory systems: the input-output relation is a power function (Stevens, 1961). The exponent varies with sense modality and also with certain other parameters such as adaptation (J. C. Stevens \& S。 S. Stevens, 1963), contrast (Horeman, 1965; Stevens, in press; Stevens 


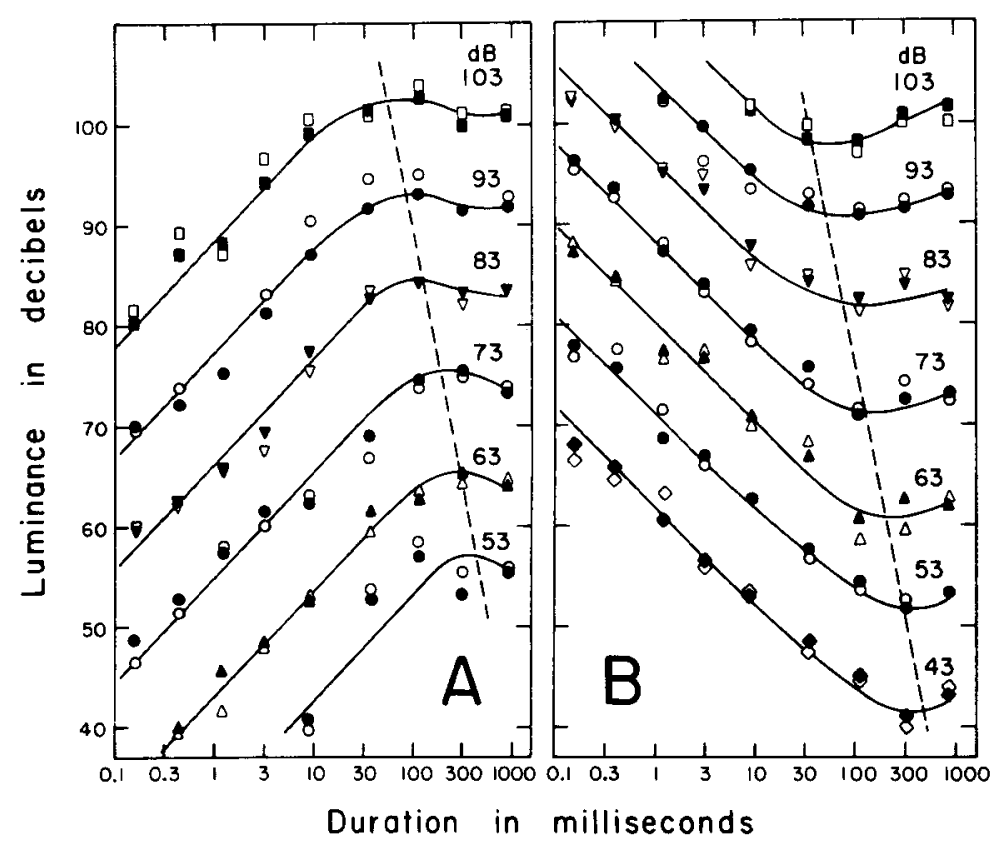

Fig. 1. Results of matching the brightnesses of various durations. The flashes were presented successively, each to a different eye. The filled points are geometric means (decibel averages) of 20 observations, 4 by each of 5 observers. The unfilled points are medians. The parameter is the peak luminance of the flash, expressed in decibels re $10^{-10} \mathrm{~L}$. For plot $\mathrm{A}$ a 1-sec. flash was matched to the shorter flashes. For plot $B$ the shorter flashes were matched to the 1-sec. flash. The curves were adjusted to reflect the operation of Bloch's law at short durations. The dashed line shows how the BrocaSulzer effect shifts to shorter durations when the luminance is raised.
\& Diamond, 1965), and, as we shall see, duration. The method of magnitude estimation was used by Raab (1962) in an experiment on the brightness of short flashes. Eighteen observers matched numbers to the apparent brightness of flashes that varied over wide ranges of duration and luminance, making up a total of 91 stimuli. Three daily sessions were required so that each stimulus could be presented once to each observer. A standard stimulus, $200 \mathrm{msec}$, at $75 \mathrm{db}$ re $10^{-10} \mathrm{~L}$, was presented at the outset and after every five trials. The observer was told to call the standard flash 10 and to assign proportional numbers to the other stimuli, depending on how bright they appeared. The observers were asked to try to ignore the differences in duration and to judge only the brightness.

Raab computed and published the medians of the judgments for each stimulus. Since numerous studies have shown that magnitude estimations tend to give log normal distributions, and since the geometric mean is a more efficient statistic than the median, the geometric means were computed from the original data kindly supplied by Professor Raab. These geometric means are plotted in Fig. 2.

The outcome of the experiment was summarized by Raab as follows: "The median numerical estimations (made relative to a standard flash) confirmed three principles: the reciprocity between luminance and duration (Bloch's law), the enhancement of brightness at about $50 \mathrm{msec}$. (Broca-Sulzer effect), and the powerlaw relation between brightness and energy (Stevens's law)." If the medians could be said to confirm those three principles, the geometric means make them doubly obvious. As with the data of Fig. 1, the curves in Fig. 2 have been fitted to the points in a manner consistent with certain restraints. Thus, for the short durations, the slope and spacing of the curves has been made to accord with Bloch's law. For the long durations, the spacing approximates the prediction of the psychophysical power law relating brightness $B$ to luminance $L$

$$
B=k\left(L-L_{o}\right) \cdot 33
$$

where $L_{o}$ is the effective threshold.

As in Fig. 1, the dashed line in Fig. 2 shows how the Broca-Sulzer hump moves to shorter durations when the luminance of the flashes increases. It will be noted that the data for flashes having peak luminances of $95 \mathrm{db}$ do not exhibit evidence of the usual brightness enhancement. The curve has therefore been dashed, but the evidence of Fig. 1 suggests that the dashed curve may in fact turn out to be more representative than the data points themselves. Since the pairs of curves for 93 and $103 \mathrm{db}$ in Fig. $1 \mathrm{~A}$ and $1 \mathrm{~B}$ show the Broca-Sulzer hump in an unmistakable manner, it seems improbable that the effect would vanish at a level intermediate between those two levels.

There is a notable similarity between Fig. 1A and Fig. 2. Both sets of data are the results of matching experiments: brightness matched to brightness for Fig. 1A, numbers matched to brightness for Fig. 2. The view that these two types of matching procedures differ in some fundamental respect seems to be contradicted by the similarity of the outcomes. In these, and in numerous other matching experiments, it has been demonstrated empirically that the number continuum is, from the point of view of the observer's behavior, essentially like any other stimulus continuum. The observer matches number to brightness much as he matches brightness to brightness, loudness to brightness (J. C. Stevens \& Marks, 1965), handgrip to brightness (J. C. Stevens, Mack, \& S. S. Stevens, 1960), and 


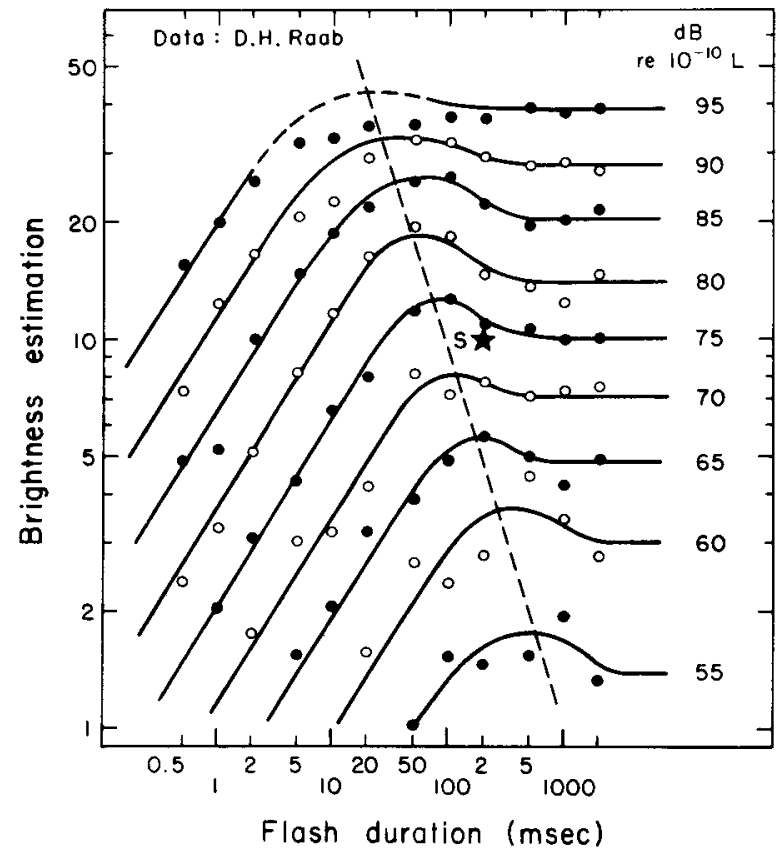

Fig. 2. Results of matching numbers to the brightnesses of flashes of various durations and luminances. Each point is the geometric mean of the judgments of 18 observers. The star $S$ des ignates the standard stimulus, called 10. Relative to this standard the observers made magnitude estimations of the brightness of the other stimuli presented in irregular order. The slanted dashed line shows how the Broca-Sulzer effect shifts with luminance. The curves were made to agree with the requirements of Bloch's law at the short durations.

length of lines to brightness (Stevens \& Guirao, 1963). The results of all these procedures have shown a reassuring transitivity. Transitivity among three continua is tested by showing that, given any two matching functions, the exponent of the third matching function can be predicted. For example, from matches between brightness and number, plus matches between number and handgrip, the exponent for the matching of handgrip to brightness can be successfully predicted.

The matching of number to brightness has produced similar results in several different laboratories (Stevens, 1953; Hópkinson, 1956; Onley, 1960; Jameson \& Hurvich, 1961).

\section{The Flash Exponent}

The brightness of stimuli whose duration is of the order of a second increases as the 0.33 pdwer of the luminance, in accordance with the equation noted above. An interesting question concerns what happens to the exponent in the Bloch region where brightness is governed, not by the peak luminance, but by the total energy in the flash. The answer hinges on the slope of the dashed line in Figs. 1 and 2. How this comes about can be seen with the aid of the geometrical model shown in Fig. 3.

If the angle $\theta$ in Fig. 3 were zero, the separation of any two constant-luminance curves in the flash region
F would be the same as the separation of the same two curves in the steady-state region $S$. As the angle $\theta$ increases, the separation $\mathrm{F}$ increases, even though the separation $\mathrm{S}$ remains constant. The relation among $\mathrm{S}, \mathrm{F}$, and $\theta$ is given by the equation

$$
1 / \mathrm{S}=1 / \mathrm{F}+\tan \theta
$$

In the $\log -\log$ coordinate system of Fig. 3, the separation $S$ is proportional to the exponent for a long flash (e.g., 1 sec.), and the separation $F$ is proportional to the exponent for a short flash (e.g., $1 \mathrm{msec}$ ). It therefore follows that for a given value of $\theta$ there is a fixed relation between the flash exponent and the steady-state exponent. All flashes of the order of 1 sec. have an exponent equal to 0.33 , and all flashes short enough for Bloch's law to hold have a larger exponent. Interestingly enough, if this theory is correct, only two values of the exponent are involved. What is here called the steady-state region is, of course, only relatively steady, for the apparent brightness of a target changes with the adaptation level of the eye (J. C. Stevens \& S。S.Stevens, 1963).

The size of the short-flash exponent can be estimated from the data in Figs. 1 and 2. The slope of the dashed lines in Fig. 1 is such that the flash exponent is about 15 per cent larger than the steady-state exponent, i.e., about 0.4 . Raab's data suggest a larger flash exponent, namely, about 0.5 . Further studies will be needed to determine the flash exponent more precisely and also to explore the intermediate durations where the Broca-

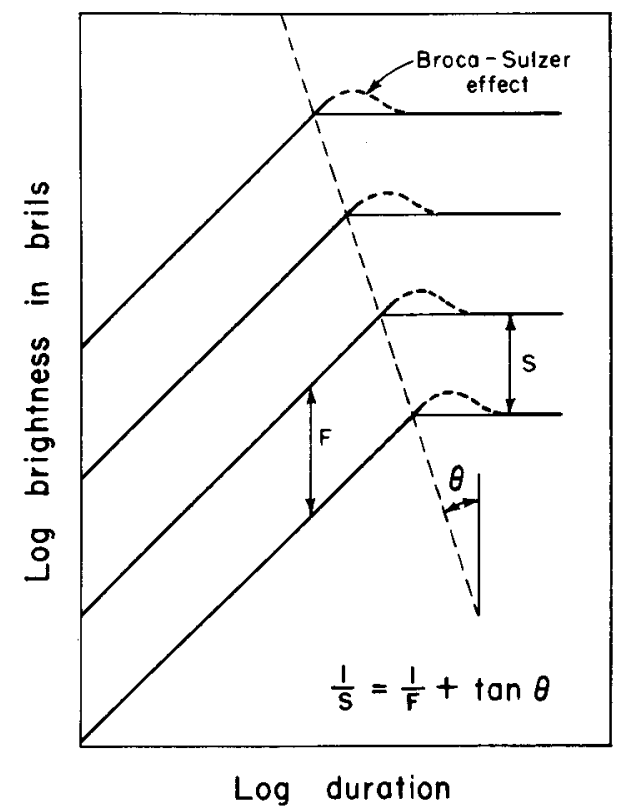

Fig. 3. Schema to show how the exponent governing the brightness of short flashes $F$ is related to the exponent for long, steadystate flashes $S$. The parameter of the curves is assumed to represent luminance, uniformly spaced in decibels. As the angle $\theta$ increases, the vertical distance $F$ increases, in accordance with the equation. In these $\log -\log$ coordinates, the distances $S$ and $F$ are proportional to the respective exponents. 
Sulzer effect should cause the function relating brightness to peak luminance to depart from a strict power function.

\section{Category Judgments}

Different degrees of constraint may occur in the various procedures by which observers can be made to assign numbers to brightnesses.In Raab's experiment, except for the value 10 which the experimenter assigned to the standard flash, the observer was free to assign any number that seemed appropriate, whole numbers, fractions, or decimals. Some experiments have shown excellent results with the standard omitted so that the observer is still less constrained than in Raab's experiment. Experience seems to endorse the tactic of leaving the observer as free from constraint as possible.

At the other extreme, the responses of the observer can be restricted to a limited number of categories, such as the whole numbers 1 to 10 . When the responses of the observer are constrained in this manner, the resulting category scale is nonlinearly related to the brightness scale obtained when the observer can make free use of the number continuum, or of some other matching continuum. The nonlinearity of the restricted category scale, which in effect forces the observer to try to partition the brightness continuum into a finite number of segments, has been demonstrated in numerous experiments. Despite those repeated demonstrations, the category procedure has been erroneously cited by Graham (1965, p. 73) as an example of the method by which the power law has been shown to hold for sensory magnitude.

A finite category scale is at best an interval scale, a scale with an arbitrary zero point, like the temperature scales Fahrenheit and Celsius. Since a constant can be added to values on an interval scale, as in the operation of changing from Celsius to Fahrenheit, the taking of logarithms of category values is a meaningless procedure. It would perhaps be better, therefore, if the ordinate of Graham's Fig. 4.7 (1965, p. 73) were made linear rather than logarithmic.

Graham seems to detect a necessity to treat numerical estimates as he would treat the results of a threshold experiment conducted by the method of constant stimuli or the method of single stimuli. He advocates a rigorous, objective behaviorism to justify that position, but, in legislating which operations shall be scientifically useful, he runs the danger of allowing rigor to verge on rigidity. Thus, according to the proscription set forth by Graham and Ratoosh (1962, p. 490), "objection may be registered against the method of averaging estimates by treating responses as though they were quantities." In other words, if ten observers estimate the apparent brightness of a series of luminances, it is not proper, say Graham and Ratoosh, to average the observers' estimates. On the other hand, it would be acceptable to the authors, if, instead of an average of the observers' estimates, an average was made of the stimulus values that led to particular estimates. For example, one could determine what stimulus, on the average, led to the response "five."

A priori theorizing in which an author pronounces do's and don't's concerning scientific method may be harmless enough. Few scientists pay much attention to philosophical dicta anyway. On the other hand, when a theoretical point of view leads to the use of experimental procedures that produce results at variance with expectations, it becomes a matter of more serious concern. It is the acknowledged eminence of Professor Graham in the field of vision that directs attention to this issue and warrants careful scrutiny of a recent experimental implementation of his unusual view concerning psychophysical measurement. The experiment in question was carried out by Lewis (1965), who, following Graham's prescription, undertook to provide "a demonstration of a psychophysical approach to category judgments."

Lewis's two observers judged the brightness of flashes that varied in duration and luminance. The procedure was not unlike that of Raab, with the crucial difference that Lewis allowed the observers to use only the integers one to ten. They could also say zero if they saw nothing. After they were told which flash to call ten, they were told, "If you were to see a flash only half as bright as the one you have just seen you would say 'five." Those familiar with category judgments will recognize an inherent contradiction in a set of instructions that asks an observer to sort 60 stimuli into ten categories and at the same time make the category numbers proportional to brightness.

In treating the data, Lewis followed Graham's injunction against averaging the numbers assigned by the observers. Instead, he averaged the stimulus values to obtain the geometric mean of the stimulus energy corresponding to each numerical response. What that procedure amounts to, in effect, is a rejection of the appropriate regression curve in favor of the less appropriate one. A similar problem arose several decades ago in the treatment of a laboratory exercise involving category judgments of lifted weights. The procedure in those days was to average the weights assigned to each category. Thurstone criticized this procedure in connection with an analogous problem of averaging data involving the category judgments of numbers of dots. He said, "At this point appears the methodological error which is involved in the wellknown Sanford weight experiment, which has been quoted by Titchener and others. If we should proceed as in the Sanford weight experiment, we should merely ascertain the average number of dots in each pile [ in each category]. These would be considered as the adjusted $\mathbf{R}$ [ stimulus] values to be plotted against the equal intervals on the $S$ [ subjective ] scale. As a matter of fact, 
such a procedure makes use of the wrong regression for this problem" (Thurstone, 1959, p. 94). A similar conclusion was voiced by Guilford $(1936, p .155)$, who said that to find the regression of the stimulus on the response "is not the best statistical procedure." Actually, if one wants to average the stimuli, the observer should be allowed to vary the stimuli as is done with the methods called magnitude production (Lane, Catania, \& Stevens, 1961; Eisler, 1962; J.C. Stevens \& Mack, 1959) and category production (Stevens \& Galanter, 1957; Stevens, 1958).

Using, then, a restricted category scale and a questionable choice of regression curve, Lewis obtained from his two observers what turned out to be conflicting results. The disagreement between the two observers led to a second experiment in which fewer stimuli were used and the observers were told, "Use only the whole numbers zero, one, two, three, or four." The final conclusion of the study was that, although Bloch's law was found to hold, neither observer gave evidence for the Broca-Sulzer phenomenon. Furthermore, "no reliable variation in the critical duration was found with changes in the luminance level." In other words, Lewis did not find the shift shown by the dashed lines in Figs. 1-3, a shift that has appeared in the results of many experiments over the past hundred years.

Having chosen to discount the evidence for the BrocaSulzer effect exhibited in Raab's study, Lewis hypothesizes that the Broca-Sulzer effect may be a contrast phenomenon, to be found only in those experiments in which brightness is matched to brightness. This hypothesis is weakened, however, not only by Raab's study, but by a similar magnitude-estimation experiment conducted in this laboratory by J.C. Stevens in which the functions obtained were similar in form to those of Raab shown in Fig. 2 .

One further comment. In championing an "objective psychology," as set forth in his theoretical paper of 1958, Graham performed the vital service of formulating a scientific challenge. By proposing that we should repudiate "the procedure of treating numerical estimates as if they were numerical data," Graham stimulates us to ask how we may measure sensation if no use is made of magnitude estimation, or kindred procedures. That challenge has led to the development of the method known as cross-modality matching, a procedure designed to avoid all use of numbers by the observer (Stevens, 1959). Two notable developments have accrued. (1) Those who may have objected to numerical estimation will now find that they do not need to use that method, for sensory scales can be satisfactorily established by cross-modality matching. Loudness provides a well documented example (Stevens, 1966). (2) A new and broader generalization seems to emerge: regardless of which continua are involved, matching is the basic process used by the observer. Any perceptual continuum can be matched to any other continuum, including the number continuum. Indeed, there may be much heuristic value in the hypothesis that all judgment reduces to the procedure of matching an aspect of one continuum to an aspect of another.

\section{References}

Aiba, T. S., \& Stevens, S. S. Relation of brightness to duration and luminance under light- and dark-adaptation. Vision Res., $1964,4,391-401$.

Bills, M. A. The lag of visual sensation in its relation to wavelength and intensity of light. Psychol. Monogr., 1920, 28, No. 5

Eisler, H. Subjective scale of force for a large muscle group. $J$ exp. Psychol., 1962, 64, 253-257.

Graham, C. H. Sensation and perception in an objective psychology. Psychol. Rev., 1958, 65, 65-76.

Graham, C. H.(Ed.), Vision and visual perception. New York:Wiley. 1965 .

Graham, C. H., \& Ratoosh, P. Notes on some interrelations of sensory psychology, perception, and behavior. In $\mathbf{S}$. Koch (Ed.) Psychology: A study of a science. Vol. 4. New York: McGrawHill, 1962. Pp. 483-514.

Guilford, J. P. Psychometric methods. New York: McGraw-Hill, 1936.

Hopkinson, R. G. Light energy and brightness sensation. Nature $1956,178,1065-1066$.

Horeman, H. W. Relation between brightness and luminance under induction. Vision Res., 1965, 5, 331-340.

Jameson, D., \& Hurvich, L. M. Complexities of perceived brightness. Science, 1961, 133, 174-179

Katz, M. S. Brief flash brightness. Vision Res., 1964, 4, 361-373.

Lane, H. L., Catania, A. C., \& Stevens, S. S. Voice level: Autophonic scale, perceived loudness, and effects of sidetone. $J$ Acoust. Soc. Amer., 1961, 33, 160-167.

Lewis, M. F. Category judgments as functions of flash luminance and duration. J. Opt. Soc. Amer., 1965, 55, 1655-1660.

Nachmias, J., \& Steinman, R. M. Brightness and discriminability of light flashes. Vision Res., 1965, 5, 545-557.

Onley, J. W. Brightness scaling of white and colored stimuli. Science, $1960,132,1668-1670$.

Raab, D. Magnitude estimation of the brightness of brief foveal stimuli. Science, $1962,135,42-43$.

Stevens, J. C., \& Mack, J. D. Scales of apparent force. J. exp. Psychol., 1959, 58, 405-413.

Stevens, J. C., Mack, J. D., \& Stevens, S. S. Growth of sensation on seven continua as measured by force of handgrip. $J$. exp. Psychol., 1960, 59, 60-67.

Stevens, y. C., \& Marks, L. E. Cross-modality matching of brightness and loudness. Proc. Nat. Acad. Sci., 1965, 54, 407-411. Stevens, J. C., \& Stevens, S. S. Brightness function: Effects of adaptation. J. Opt. Soc. Amer., 1963, 53, 375-385.

Stevens, S. S. On the brightness of lights and the loudness of sounds. Science, 1953, 118, 576. (Abstract)

Stevens, S. S. Problems and methods of psychophysics. Psychol. Bull., 1958, 55, 177-196.

Stevens, S. S. Cross-modality validation of subjective scales for loudness, vibration, and electric shock. J. exp. Psychol., 1959, 57, 201-209.

Stevens, S. S. Psychophysics of sensory function. In W. A. Rosenblith (Ed.), Sensory communication. Cambridge, Mass.: The M. I. T. Press, 1961. Pp. 1-33.

Stevens, S. S. Matching functions between loudness and ten other continua. Percept. \& Psychophys., 1966, 1, 5-8.

Stevens, S. S. Power-group transformations under glare, masking. and recruitment. J. Acoust. Soc. Amer., in press.

Stevens, S. S., \& Diamond, A. L. Effect of glare angle on the brightness function for a small target. Vision Res., 1965, 5, 649-659.

Stevens, S. S., \& Galanter, E. H. Ratio scales and category scales for a dozen perceptual continua. J. exp. Psychol., 1957, 54, $377-411$.

Stevens, S. S., \& Guirao, M. Subjective scaling of length and area and the matching of length to loudness and brightness. $J$. exp. Psychol., 1963, 66, 177-186.

Thurstone, L. L. The measurement of value, Chicago: The University of Chicago Press, 1959.

(Received in the Editorial Office February 2, 1966.) 